\title{
Mapotí: aplicativo informativo sobre política
}

\author{
Mapotí: politics informational app
}

Artur Lima Botelho, Isabela Oliveira Mosquini

aplicativo, política, experiência do usuário, interface do usuário, informação

Objetiva-se demonstrar os principais aspectos do projeto, o aplicativo Mapotí , desenvolvido para democratizar o acesso da população à informações sobre políticos candidatos e eleitos. $O$ projeto teve como inspiração os Objetivos de Desenvolvimento Sustentável da ONU, mais especificamente a diretriz 16: Paz, Justiça e Instituições Eficazes, e a compreensão de que todas as mudanças políticas, econômicas e sociais de um país precisam ser realizadas coletivamente, sendo a política o meio mais eficaz de cobrança e participação pela mudança da realidade. Foram elaborados desde os primeiros conceitos do aplicativo ao seu protótipo final, abordando diversos conhecimentos sobre UX/UI. O resultado alcançado foi um protótipo navegável que representa com fidelidade as ideias centrais do aplicativo: um ambiente que possibilite a pesquisa e o acesso à informações sobre pessoas políticas e partidos com facilidade e rapidez.

app, politics, user experience, user interface, information

The objective is to demonstrate the main aspects of the project, the app Mapotí, developed to democratize the population's access to information about candidates and elected politicians. The project was inspired by the UN Sustainable Development Goals, more specifically the guideline 16: Peace, Justice and Effective Institutions, and the understanding that all political, economic and social change need to be handled collectively, being politics the most effective way of participating in the change of reality. The project was elaborated from the first concepts of the app to its final prototype, covering various knowledges of UI/UX concepts. The result achieved was a navigable prototype that faithfully represents the central ideas of the app: an environment that enables research and access to information about political people and parties, easily and quickly.

\section{Introdução}

\section{Contexto}

O projeto foi desenvolvido nas disciplinas do Projeto Digital ofertadas pelo Design UFSC e buscou a construção de um aplicativo mobile sobre política. Ao longo dos processos, decidiu-se que o aplicativo trataria da divulgação de informações sobre políticos e partidos políticos à comunidade, por meio de uma interface simples e objetiva. 


\section{Metodologia do projeto}

A metodologia aplicada foi baseada nos Fundamentos da ISO 92412011 - Projeto Centrado no Ser Humano para Sistemas Interativos. Trata-se de uma abordagem:

"que objetiva tornar os sistemas utilizáveis e úteis, dando ênfase aos usuários, suas necessidades e exigências, pela aplicação de conhecimentos e as técnicas de usabilidade e fatores

humanos/ergonomia. Esta abordagem aumenta a eficácia e a eficiência, aprimora o bem-estar do ser humano, a satisfação do usuário, a acessibilidade e a sustentabilidade; e neutraliza possíveis efeitos adversos do seu uso na saúde, na segurança e no desempenho" (Associação Brasileira de Normas Técnicas, 2011, p.3).

Assim, o projeto foi desenvolvido sobre os pilares da pesquisa e análise, síntese e conceito, estruturação, avaliação, e design visual. Quando se trata de um projeto centrado no usuário, esses pilares são interdependentes.

\section{Pesquisa e análise de dados}

\section{Contexto inicial do projeto}

As instituições políticas, juntamente com a participação da população, devem ser detentoras do poder de mudança, por isso foi abordada diretriz 16: Paz, Justiça e Instituições Eficazes da ONU, cujo objetivo é "promover sociedades pacíficas e inclusivas para o desenvolvimento sustentável, proporcionar o acesso à justiça para todos e construir instituições eficazes, responsáveis e inclusivas a todos os níveis" (Organização das Nações Unidas, 2015).

O aplicativo, então, tem como propósito facilitar o acesso dos cidadãos à informações como gastos, votações e histórico de políticos candidatos e eleitos e seus partidos, proporcionando aos que o utilizam a possibilidade de se manterem atualizados sobre o que tem sido feito por seus representantes para incentivar uma maior participação política da sociedade. Logo, os conceitos norteadores utilizados foram os de democratização, acessibilidades e participação.

\section{Análise de similares}

Com base no contexto inicial do projeto, realizou-se a análise de similares, útil para, como indicado na ISO 9241-210 "fornecer informações sobre uma gama de questões do contexto, incluindo deficiências e níveis de referência de performance e satisfação" (Associação Brasileira de Normas Técnicas, 2011,p.15). A análise permitiu a percepção de limitações e pontos fracos que poderiam ser evitados ao projetar o aplicativo, assim como pontos fortes a serem considerados.

Os similares analisados foram os aplicativos Meu Deputado e Poder do Voto, resultando no desenvolvimento de quadros comparativos de funcionalidades: 
Figura 1 - Quadro comparativo de funcionalidades dos similares.

\begin{tabular}{|c|c|c|}
\hline Funcionalidades & MD & PV \\
\hline Login através do Facebook & $\checkmark$ & $\checkmark$ \\
\hline Acesso anônimo & $\checkmark$ & X \\
\hline Ferramenta de busca por nome & X & X \\
\hline Ferramente de busca por partido & X & $\checkmark$ \\
\hline Ferramenta de busca por localização & X & $\checkmark$ \\
\hline Filtros de busca por estado/partido & $\checkmark$ & $\checkmark$ \\
\hline Filtro de busca por data & $\checkmark$ & X \\
\hline Organização por ordem alfabética & $\checkmark$ & $\vee$ \\
\hline Perfil do político & $\checkmark$ & $\checkmark$ \\
\hline Compartilhamento & $\checkmark$ & X \\
\hline Seguir político & $\checkmark$ & X \\
\hline Comparação entre políticos & $\checkmark$ & X \\
\hline Notificações & X & X \\
\hline Mensagem & $\mathrm{x}$ & $\vee$ \\
\hline Redirecionamento para leis na íntegra & $\checkmark$ & $\checkmark$ \\
\hline Redirecionamento para e-mail & $\checkmark$ & X \\
\hline Avaliações & $\mathrm{x}$ & X \\
\hline Fale conosco & $x$ & $\checkmark$ \\
\hline Imagem & $\checkmark$ & $\checkmark$ \\
\hline Gráfico & $\checkmark$ & X \\
\hline Descrição do perfil & X & X \\
\hline Descrição das leis e votações & $\checkmark$ & $\checkmark$ \\
\hline Descrição do aplicativo & $\checkmark$ & X \\
\hline Partido do político & $\checkmark$ & $\vee$ \\
\hline Estado do político & $\checkmark$ & $\checkmark$ \\
\hline E-mail do político & $\checkmark$ & X \\
\hline Despesas do político & $\checkmark$ & X \\
\hline Presença & $\checkmark$ & X \\
\hline Votações & $\checkmark$ & $\checkmark$ \\
\hline Ranking dos gastos mensais & $\checkmark$ & X \\
\hline Central de ajuda & X & $\vee$ \\
\hline
\end{tabular}

Assim, foram identificadas funcionalidades a serem implementadas no aplicativo, como os gráficos de gastos do app Meu Deputado, por exemplo, e outras características negativas evitáveis, como a ausência de informações completas sobre alguns políticos.

Finalmente, o principal diferencial implementado em relação aos similares, foi a possibilidade de pesquisar informações sobre qualquer pessoa política, sendo elas vereadores, prefeitos, deputados estaduais e federais, senadores e presidentes, mantendo uma boa arquitetura e hierarquia das informações. 


\section{Entrevistas}

A etapa de entrevistas foi realizada objetivando uma conversa com o usuário. Para realizá-las, partiu-se da definição de entrevistas semi estruturadas, sendo elas, de acordo com o livro Design e Interação: além da interação humano-computador (Preece et alii, 2005), as que não são rígidas como uma entrevista estruturada nem livres como uma não-estruturada. Elas combinam perguntas abertas e fechadas guiadas por um roteiro que se inicia com perguntas pré-planejadas e é estendida até que o entrevistado não possua nenhuma informação nova.

Formularam-se perguntas objetivas a seis usuários contatados de forma remota, por Instagram e Whatsapp, e entrevistados por meio de plataforma online, pelo Google Meet. As entrevistas foram gravadas para consulta posterior e os dados coletados foram utilizados para desenvolver os gráficos de comportamento. Por meio dos gráficos, percebeu-se algumas características norteadoras.

A maior parte dos entrevistados é jovem, com idades entre 19 e 21 anos, sendo a grande parte estudantes universitários, informações que expõem uma possível facilidade com tecnologias e uso regular de aplicativos. Portais de notícia, redes sociais e jornais são os principais meios de acesso a notícias e todos os entrevistados utilizam o celular como principal fonte de pesquisa, o que indica que o uso de um aplicativo de consulta política poderia ser incorporado no dia-a-dia dos entrevistados. Algumas pessoas também demonstraram interesse em ter acesso a informações sobre lideranças sociais e não somente políticos eleitos. Ao final da entrevista, alguns disseram que gostariam que o design do aplicativo fosse simples e fácil de entender e navegar e nenhum dos entrevistados conhecia aplicativos com o mesmo tema do projeto.

\section{Personas}

Após as entrevistas, geração e análise dos gráficos de comportamento, desenvolveram-se 2 personas para o projeto, sendo uma primária e outra secundária. 
Figura 2 - Persona primária.

Persona Primária

Fernanda Souza

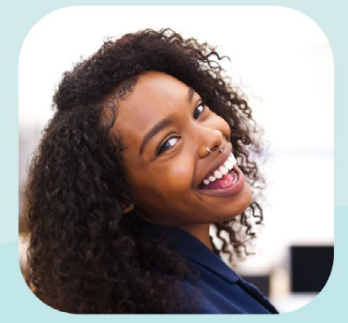

Idade: 21 anos

Localização: São Paulo Ocupação: Estudante Nível Educacional:

Ensino Superior Incompleto
"Preciso me manter informada e lutar pelos meus direitos."

\section{Objetivo Final}

Consultar informações sobre políticos candidatos e eleitos de diferentes perfis.

Objetivos da Experiência

- Sentir-se informada e apta a opinar

- Sentir facilidade para acessar informações

- Sentir segurança em relação às fontes dos dados

Figura 3 - Persona secundária.

Persona Secundária
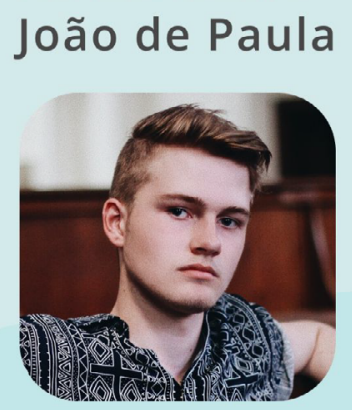

Idade: 23 anos

Localização: Uberaba Ocupação: Estudante Nível Educacional: Ensino Superior Incompleto
"Quero decidir em quem votar com praticidade!"

Objetivo Final

Decidir em quem votar.

\section{Objetivos da Experiência}

- Sentir que foi ajudado no processo de pesquisa

- Sentir facilidade para acessar

informações

- Sentir que encontrou o que

queria com rapidez

Assim como citado no livro O Guia para Projetar UX, "personas bem arquitetadas geralmente são usadas como um ponto de interação sempre que uma pergunta ou dúvida surge sobre como os aspectos do projeto devem ser arquitetados" (Unger; Chandler, 2009) e assim foram utilizadas na execução deste projeto. 


\section{Síntese}

Com base nas entrevistas e considerando as personas desenvolvidas, foram definidas histórias de usuários e requisitos funcionais e de conteúdo, que colaboraram para o desenvolvimento de ferramentas fundamentais ao aplicativo, sendo cada requisito baseado em uma história de usuário.

Figura 4 - Requisitos de projeto.

\begin{tabular}{|c|c|c|}
\hline Requisito & Funcionalidade & Conteúdo \\
\hline $\begin{array}{l}\text { Quero encontrar } \\
\text { com facilidade o } \\
\text { político a ser pesquisado }\end{array}$ & $\begin{array}{l}\text { Organização por } \\
\text { categorias }\end{array}$ & $\begin{array}{l}\text { Linguagem } \\
\text { acessível }\end{array}$ \\
\hline $\begin{array}{l}\text { Quero encontrar } \\
\text { informações com rapidez }\end{array}$ & Ferramenta filtrar & Gráficos \\
\hline $\begin{array}{l}\text { Quero saber no que meu } \\
\text { candidato tem votado }\end{array}$ & $\begin{array}{c}\text { Direcionamento } \\
\text { para leitura da lei } \\
\text { na íntegra }\end{array}$ & Leis e votações \\
\hline $\begin{array}{l}\text { Quero acompanhar } \\
\text { a atividade de } \\
\text { alguns políticos }\end{array}$ & $\begin{array}{l}\text { Seguir perfil } \\
\text { Login do usuário }\end{array}$ & $\begin{array}{l}\text { Informações } \\
\text { atualizadas }\end{array}$ \\
\hline $\begin{array}{l}\text { Quero poder buscar por } \\
\text { partidos e conseguir } \\
\text { informações }\end{array}$ & $\begin{array}{l}\text { Ferramenta } \\
\text { de busca } \\
\text { Ferramenta } \\
\text { de filtro }\end{array}$ & $\begin{array}{l}\text { História do partido } \\
\text { Histórico de } \\
\text { participação }\end{array}$ \\
\hline $\begin{array}{l}\text { Quero conhecer } \\
\text { novos candidatos }\end{array}$ & $\begin{array}{l}\text { Ferramenta } \\
\text { de busca }\end{array}$ & -- \\
\hline $\begin{array}{l}\text { Necessito estar apta a } \\
\text { escolher um candidato }\end{array}$ & $\begin{array}{l}\text { Ferramenta de } \\
\text { comparação }\end{array}$ & $\begin{array}{l}\text { Informações } \\
\text { completas e } \\
\text { atualizadas }\end{array}$ \\
\hline $\begin{array}{l}\text { Compartilhar o } \\
\text { perfil de um político } \\
\text { com outras pessoas }\end{array}$ & $\begin{array}{l}\text { Ferramenta } \\
\text { compartilhar }\end{array}$ & -- \\
\hline
\end{tabular}

\section{Estruturação}

Iniciou-se então a estruturação do aplicativo. Houve o cuidado de organizar as informações da maneira mais intuitiva possível. A arquitetura da informação pode ser conferida abaixo, onde também já está definido o design da navegação. 
Figura 5 - Mapa do design da navegação.

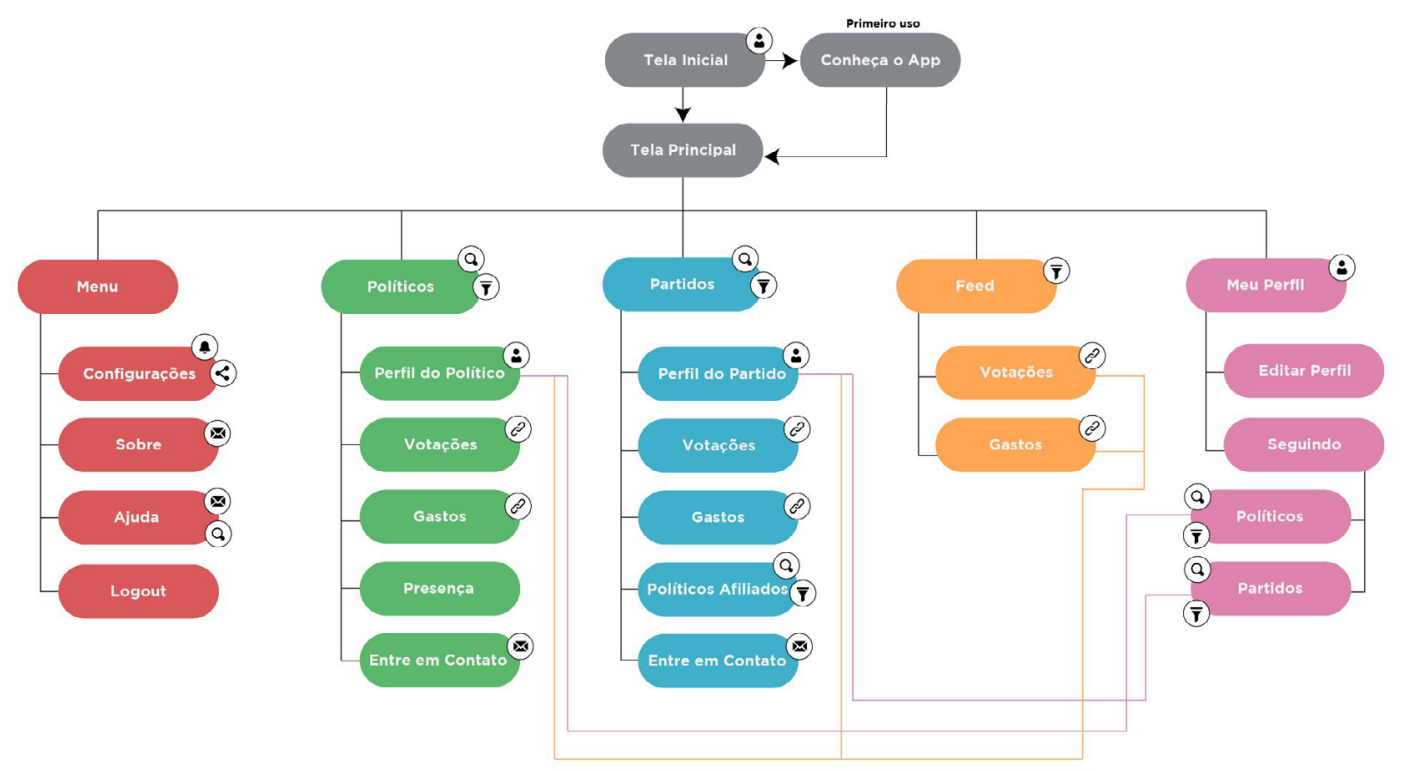

\section{Wireframes}

A partir da estruturação, iniciou-se a elaboração dos wireframes. Foram desenvolvidas 19 telas que demonstram a organização das informações. Elas foram pensadas para guiar as próximas decisões do projeto e também para serem avaliadas nos testes de usabilidade. Algumas delas podem ser conferidas abaixo.

Figuras 6, 7 e 8 - Telas de políticos, feed e partidos.

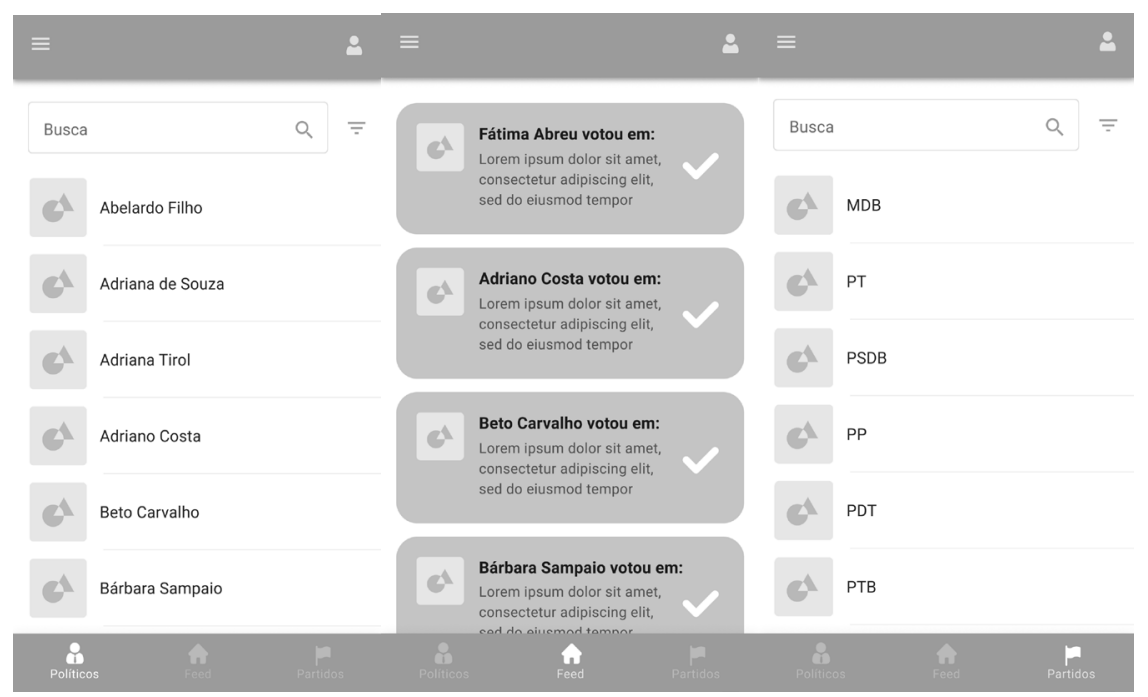


Figuras 12, 13 e 14 - Telas de votações, gastos e contato do político.

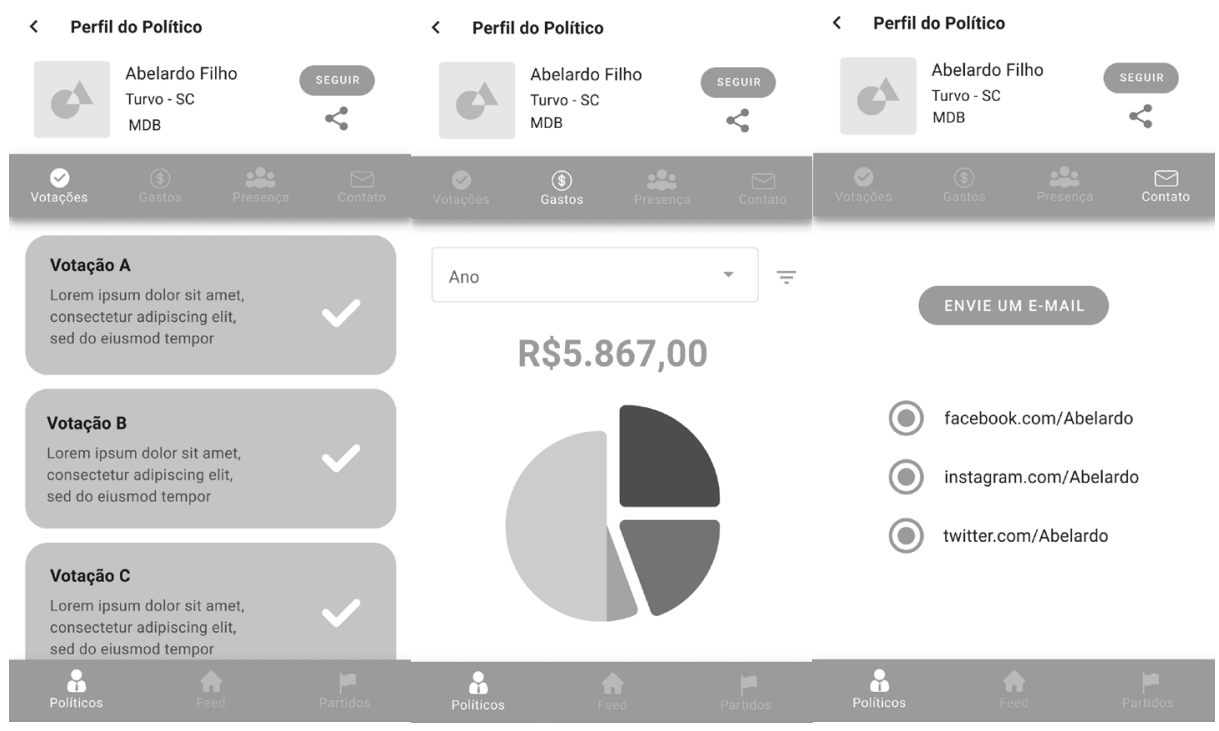

\section{Design Visual}

Para decidir o design do aplicativo, realizou-se inicialmente o moodboard da Persona 1, com o objetivo de compreender melhor como se comporta, do que gosta, suas referências de estilo e o que vivencia no dia a dia. Em seguida, desenvolveu-se o conceito de confiança, outro norteador. Por fim, gerou-se a alternativa descrita a seguir.

\section{Definição da Paleta de Cores}

Definiu-se que a paleta traria a ideia de diversidade, representada pela relação de cores quentes e frias. Foi considerado que "as cores constituem estímulos psicológicos para a sensibilidade humana, influindo no indivíduo para gostar ou não de algo, para negar ou afirmar, para se abster ou agir" (Farina, M., Perez, C., Bastos, D., 2011). A paleta também foi definida para garantir a legibilidade e leiturabilidade.

\section{Tipografia}

A fonte Open Sans possibilita um bom uso por possuir uma família extensa e variada, contribuindo para uma melhor hierarquia das informações. Além disso, é uma fonte recomendada para web e mobile, de acordo com seu criador Steve Matteson, que também a considera "desenvolvida para leitura ergonômica de textos em telas", (Matteson, 2021).

\section{Diagramação}

Foi utilizado o grid de quatro colunas, com margem $20 \mathrm{px}$ e gutter $15 \mathrm{px}$, pensando em garantir boa distribuição das informações e possibilitar um design responsivo. Definiu-se tamanhos e espessuras diferentes para cada tipo de texto e priorizou-se o alinhamento à esquerda para 
boa leiturabilidade. As imagens foram mantidas em suas cores e edições originais, buscando o melhor reconhecimento possível do político pelo usuário, e as margens foram arredondadas.

Ícones

Os ícones utilizados foram escolhidos pensando no fácil reconhecimento pelo usuário, por serem comuns a outros aplicativos e na web. Além disso, optou-se por desenhos simplificados, para uma boa visualização.

\section{Prototipação, testes de usabilidade e resultado final}

Após decididas as diretrizes visuais, foi desenvolvido o protótipo navegável, com os resultados finais obtidos e a construção completa de algumas telas do aplicativo. A partir do protótipo, foram realizados testes de usabilidade com as mesmas pessoas entrevistadas em etapas anteriores. Os testes foram realizados de maneira online, onde foi disponibilizado o protótipo para uso em seus próprios celulares. Pelos testes, foi possível perceber que as ações eram facilmente executadas e a interface não gerava dúvidas ao usuário, cumprindo com o propósito de boa usabilidade. O protótipo pode ser acessado pelo link abaixo, assim como a visualização das telas a seguir.

https://www.figma.com/proto/KQKOeYzphzz6UpXFZHiLBI/Wireframes-Projeto-Digital---Artur-eIsabela?node-id=211\%3A2699\&scaling=min-zoom\&page-id=108\%3A3484.

Figuras 15, 16 e 17 - Telas de políticos, feed e partidos.

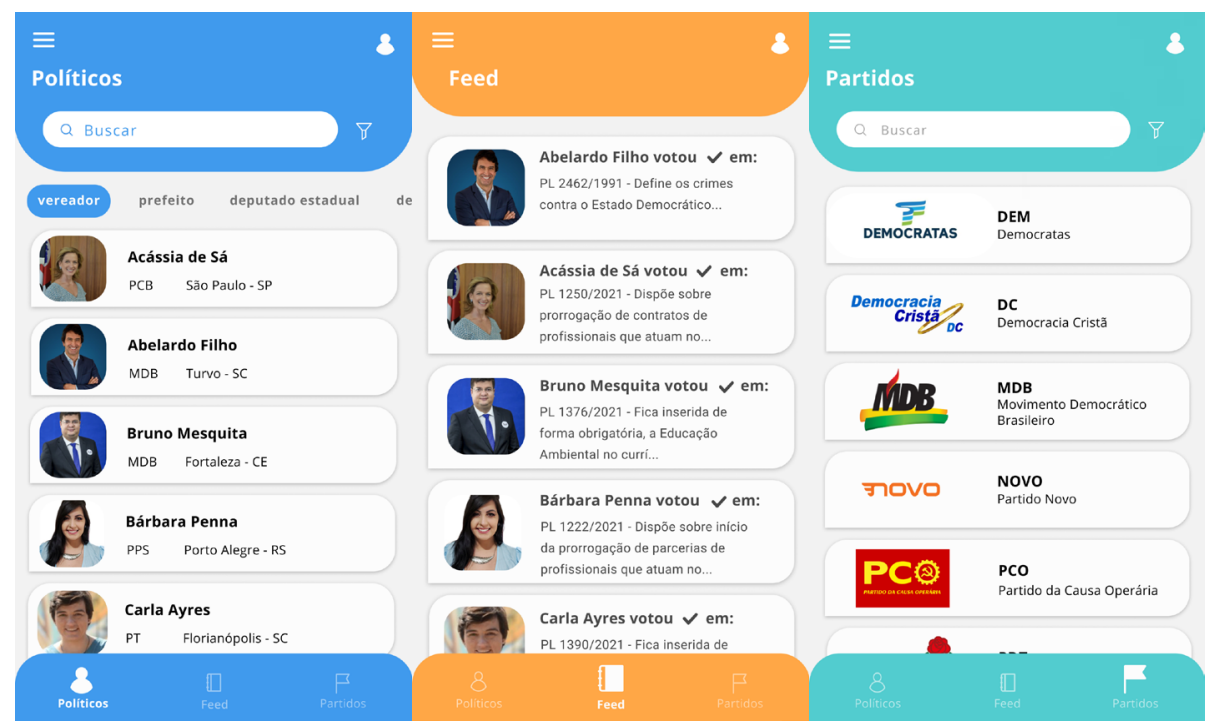


Figuras 18, 19 e 20 - Telas de votações, presença e contato do perfil do político.

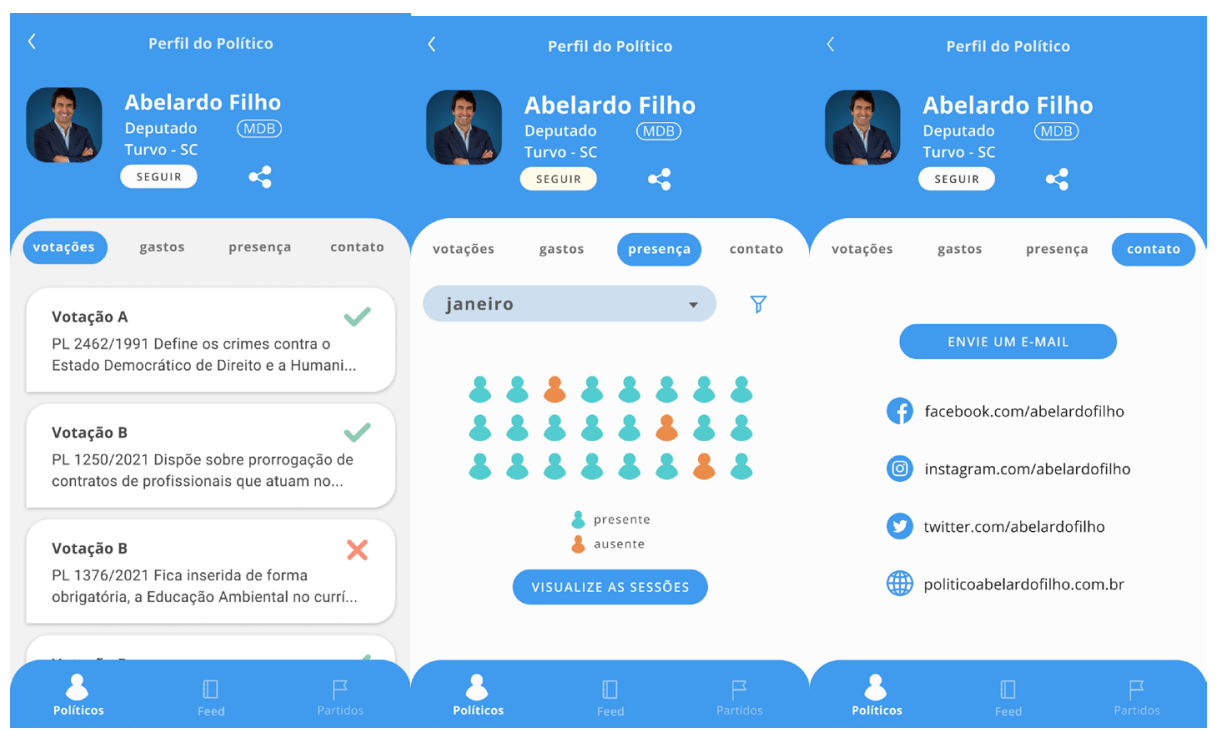

\section{Considerações finais}

No início do projeto, visou-se aspirar os usuários à mudança coletiva, pautada na compreensão de que problemas complexos exigem mudanças profundas nas estruturas sociais. Para a execução do aplicativo, almejou-se alcançar um resultado o mais próximo possível dos conceitos norteadores: acessibilidade, democratização e participação.

O resultado do protótipo foi positivo pois alcançou as expectativas de usabilidade. Participantes do projeto que atuaram nas etapas de entrevista e testes avaliaram o aplicativo positivamente, demonstrando interesse em sua existência.

Por fim, acredita-se que o projeto tem grande potencial para ser continuado, porque traz à tona a importância do envolvimento das pessoas na política, participando ativamente de decisões e fiscalizando políticos a fim de gerar mudanças significativas para a sociedade.

\section{Referências}

Organizações das Nações Unidas Brasil. Os Objetivos de Desenvolvimento Sustentável no Brasil: Paz, Justiça e Instituições Eficazes. Disponível em https://brasil.un.org/pt-br/sdgs/16

Preece, J., Rogers,Y.,Sharp, H. (2005). Design de Interação: além da interação humanocomputador. Porto Alegre: Bookman.

Associação Brasileira de Normas Técnicas (2011). NBR ISO 9241-210: Projeto centrado no ser humano para sistemas interativos. Rio de Janeiro.

Farina, M., Perez, C., Bastos, D. (2011). Psicodinâmica das Cores em Comunicação. 6. ed. São Paulo: Blucher. 
Matteson, Steve. Open Sans Hits 10 trillion. 2021. Disponível em: https://mattesontypographics.com/blog/f/open-sans-hits-10trillion?blogcategory=Web+Typography.

Unger, R., Chandler, C. (2009). O Guia Para Projetar UX. Florianópolis: Alta Books.

\section{Sobre os autores}

L. Botelho, Artur, UFSC, Brasil <arturlbot@gmail.com>

O. Mosquini, Isabela, UFSC, Brasil <isabelaomosquini@gmail.com> 